\title{
Network Security Risk Assessment Based on Item Response Theory
}

\author{
Fangwei Li \\ Chongqing Key Lab of Mobile \\ Communications Technology \\ Chongqing University of Posts \\ Telecommunications \\ Chongqing, China \\ lifw@cqupt.edu.cn \\ Jiang Zhu \\ Chongqing Key Lab of Mobile \\ Communications Technology \\ Chongqing University of Posts \\ Telecommunications \\ Chongqing, China \\ zhujiang@cqupt.edu.cn
}

\author{
Qing Huang \\ Chongqing Key Lab of Mobile \\ Communications Technology \\ Chongqing University of Posts \\ Telecommunications \\ Chongqing, China \\ huangq46@163.com \\ Zhuxun Peng \\ Chongqing Key Lab of Mobile \\ Communications Technology \\ Chongqing University of Posts \\ Telecommunications \\ Chongqing, China \\ pengzhux@163.com
}

\begin{abstract}
Owing to the traditional risk assessment method has onesidedness and is difficult to reflect the real network situation, a risk assessment method based on Item Response Theory (IRT) is put forward in network security. First of all, the novel algorithms of calculating the threat of attack and the successful probability of attack are proposed by the combination of IRT model and Service Security Level. Secondly, the service weight of importance is calculated by the three-demarcation analytic hierarchy process. Finally, the risk situation graph of service, host and network logic layer could be generated by the improved method. The simulation results show that this method can be more comprehensive consideration of factors which are affecting network security, and a more realistic network risk situation graph in real-time will be obtained.
\end{abstract}

\section{Categories and Subject Descriptors}

D.4.6 [Operating Systems]: Security and Protection; C.2.3 [Computer-Communication Networks]: Network Operations-Network monitoring

\section{General Terms}

Security

\section{Keywords}

IRT, hierarchical model, risk assessment, network security

\section{INTRODUCTION}

Network security situation awareness [7] is a new security technology which is based on the analysis of historical data and the detection of current network security status. Network security situation assessment is the important bond between the obtainment of situation elements and the prediction of trend in network security situation awareness. To solve wireless network risk evaluation problems, a four-layer of wireless network risk assessment mechanism was proposed by the characteristics of different configuration [10]. Concurrently, some traditional assessment methods only considered the influence of vulnerability by scanning in the network but not safety measures [1,13]. In fact, the threat of network is the inherent vulnerability threat after taking safety measures. Moreover, the threat of attack is defined too broad in most methods of risk assessment $[9,5]$.

This paper puts forward a method of combining item response theory with the hierarchical network risk assessment model. It will solve some problems. Firstly, the lack of objectivity in the successful probability of attack will be improved by the system information, Common Vulnerability Scoring System (CVSS) [2,8] and the safety measures. Next, the concept of attack ability is proposed by item response theory and the discrimination of threat of attack is improved. Following this, with the three-demarcation analytic hierarchy process [12] to calculate the service weight of importance, the accuracy will be improved. The last, the risk situation graph of service, host, and network logic layer are draw by analyzing each layer of the risk situation.

\section{NETWORK SECURITY RISK ASSESSMEN- T BASED ON ITEM RESPONSE THEO- RY}

Item response theory $[4,3,11]$ is also called the latent trait theory, widely used in pedagogy and originated by the American psychological statistician Frederic M. Lord. It is a kind of method by estimating the tester ability and linking 


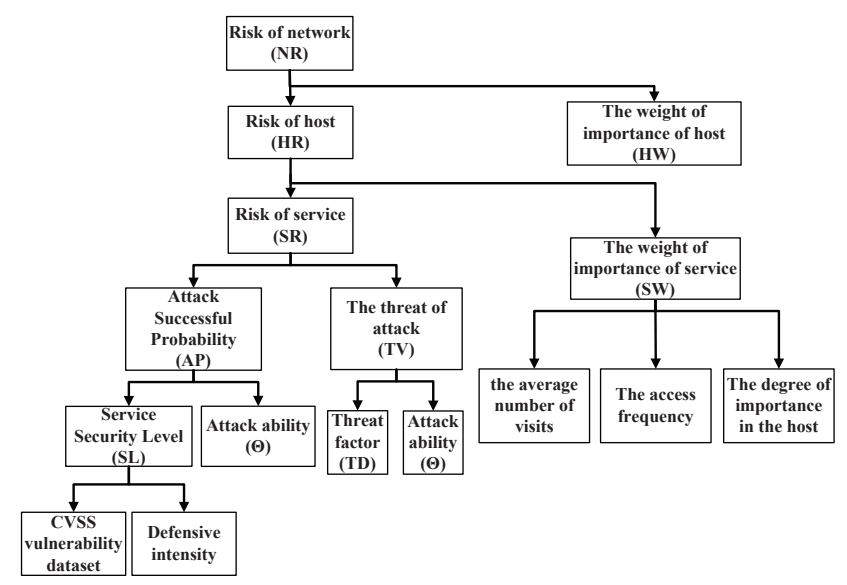

Figure 1: The Real-time Network Security Risk Assessment Framework

the reaction probability of tester for each project.

\subsection{The Real-time Network Security Risk As- sessment Framework}

In order to scientifically evaluate the network security and effectively manage risk accident, some measures should be taken. Network security situation risk is displayed by risk situation graph, and then we can focus on protecting high risk of service or host. As the size of network expands in real life constantly, the network system(System), from the bottom to up, is divided into vulnerability logic layer (Vulnerability), service logic layer (Service), host logic layer (Host) and network logic layer (Network), and is given as follows:

$$
\text { System }=R_{V} \cup R_{S} \cup R_{H} \cup R_{N}
$$

where $R_{V}=\cup R_{v_{i}}$ is a set of vulnerability logic layer properties which reflects the degree of difficulty to attack service by the existing vulnerabilities in the services the complexity of attack and the conditions of attack.

where $R_{S}=\cup R_{s_{i}}$ is a set of service logic layer properties which reflects the risk situation of attacked service by service category, quantity and degree of importance in the hosts.

where $R_{H}=\cup R_{h_{i}}$ is a set of host logic layer properties which reflects the risk situation of working host by host category, quantity and degree of importance in the network.

where $R_{N}$ is a set of network logic layer properties which represents the polymerization of logical layer.

The real-time network security risk assessment framework is shown in Figure 1.

Based on the alarm information of IDS, target network topology and vulnerability information, combining with the rule base, the risk situation is evaluated from service logic layer to network logic layer.

The following are four basic definitions in the process of assessing.

Definition 1. Attack alarm (A): The behaviors which can trigger IDS alarm information are called attack alarm. It can be expressed as $\mathrm{A}=\{\mathrm{ID}$, Time, Type, SIP, DIP, SP, $\mathrm{DP}\}$, where ID is serial number of attack event, Time is its time occurring, Type is the type of attack activity, SIP and SP are IP address and port number of attack source respectively, DIP and DP are IP address and port number of attack destination respectively.

Definition 2. The service weight of importance (SW): SW reflects the degree of important service in the host, which depends on effect from the average number of visits, access frequency and degree of importance in the host. $S W_{i}$ is the $i$ service weight of importance and is expressed as follows:

$$
S W_{i}=\frac{S I_{i}}{\sum_{k=1}^{n} S I_{k}}
$$

where $S I_{i}$ is the importance of $i$ service, $n$ is the total number of running services in the host.

Definition 3. Attack Successful Probability (AP): Attack Successful Probability is the successful possibility of attack which utilizes service vulnerabilities and performs dangerous activities.

Definition 4. Defensive intensity (I): Defensive intensity is the degree of obstruction to the security protection measures taken on attack.

\subsection{The Threat of Attack Algorithm Based on Item Response Theory}

The threat of network attack is mainly divided into two parts: the one is for the user permissions, including fake user identity legitimacy and illegally elevate permissions to steal or distort the information of server; the other is a malicious traffic shock for the network function, which uses a large number of service requests to consume service resources, as a result, the server is difficult or impossible to handle the legitimate user requests. The threat of network attack adopts the algorithms of [9] and [5], which uses the threat factor to define the threat of attack on service, by distinguishing port scan attack, denial of service, elevated privileges and the remote user attack to define all kinds of attack. Then the threat factor is regarded as a major factor in judging the threat of attack.

However, we consider the different behaviors of attack in the same type of attack, they may be different in the threat of attack. In order to improve the discrimination of the traditional threat of attack, the concept of attack ability is proposed by item response theory and a new threat of attack formula is redefined as formula (4).

$$
T V_{i}=10^{T F_{i}} \frac{\theta_{i}+4}{7}
$$

where $\theta_{i}$ is the ability of $i$ attack which is determined by the parameter estimation from single parameter logistic function and attack response matrix; $T F_{i}$ is the threat factor of $i$ attack which is separately quantized as digits $1 \sim 3$.

\subsection{Attack Successful Probability Algorithms Based on Item Response Theory}

The execution of a successful attack and some conditions are inseparable, for instant, special ports are opened, the security defects are found in certain positions, the vulnerabilities can be exploited and so on. When these conditions are not satisfied or partially satisfied, Attack Successful Probability will be greatly reduced. For [13] does not consider the impact of Defensive intensity and attack ability, the Attack Successful Probability is redefined depending on the item response theory. 
Definition 5. Service Security Level (SL): the degree of obstruction which is appeared after attacking is referred to Service Security Level. It is constituted by the vulnerability information (C) and Defensive intensity (I). The vulnerability information is determined by CVSS, as shown in Table 1, and the formula is defined as $C=\frac{A C+A V+A U}{3}$. Defensive intensity is listed in Table 2 .

According to the above analysis, Service Security Level is defined as formula (5), at the same time, combined with a single parameter Logistic model of item response theory, Attack Successful Probability is put forward as formula (6).

$$
\begin{gathered}
S L_{j}=3\left(\lambda_{1} C_{j}+\lambda_{2} I_{j}-2\right) \\
A P_{i j}=\left\{1+\exp \left[-D\left(\theta_{i}-S L_{j}\right)\right]\right\}^{-1}
\end{gathered}
$$

where $\lambda_{1}$ and $\lambda_{2}$ show that the vulnerability and security measures accounts for the proportion in service safety respectively, through the past experience, $\lambda_{1}=0.4$ and $\lambda_{2}=0.6 ; \theta_{i}$ indicates that the ability of $i$ attack; $D=1.702$ is a constant. With the passage of time, new attacks will be detected, the original attack response matrix will be changed and the attack ability will be updated. Thus, the threat of attack and Attack Successful Probability can realize the real-time updating.

Table 1: The Information of CVSS

\begin{tabular}{|c|c|c|}
\hline \multirow{2}{*}{ CVSS Index } & Index Description & Difficulty Level \\
\hline \multirow{2}{*}{$\begin{array}{c}\text { AccessComplexity } \\
(\text { AC) }\end{array}$} & high (H) & 3 \\
\cline { 2 - 3 } & medium (M) & 2 \\
\cline { 2 - 3 } & low (L) & 1 \\
\hline \multirow{2}{*}{$\begin{array}{c}\text { AccessVector } \\
(A V)\end{array}$} & remote (R) & 3 \\
\cline { 2 - 3 } & adjacent (A) & 2 \\
\cline { 2 - 3 } Authentication & local (L) & 1 \\
\cline { 2 - 3 }$($ AU) & multiple (M) & 3 \\
\cline { 2 - 3 } & single (S) & 2 \\
\hline
\end{tabular}

\section{HIERARCHICAL NETWORK RISK AS- SESSMENT MODEL}

\subsection{The Factor of Risk Weight}

According to the factors that affect the service performance, the importance of service is mainly decided by the average number of visits, access frequency, degree of importance in the host and is calculated by the algorithm of three-demarcation analytic hierarchy process. We establish a process of calculating weight step by step from index layer to target layer.

Table 2: The Information of Defensive Intensity

\begin{tabular}{|c|c|c|}
\hline Defend & Defensive Measures Description & Intensity \\
\hline Low & No measure & 1 \\
\hline Medium & Defensive measures is not obvious & 2 \\
\hline High & Targeted defense measures & 3 \\
\hline
\end{tabular}

a) Establish comparison matrix

$$
\mathrm{z}_{i j}=\left\{\begin{array}{cc}
2 & \text { when } \mathrm{z}_{i} \text { is more important than } \mathrm{z}_{j} \\
1 & \text { when } \mathrm{z}_{i} \text { is the same important as } \mathrm{z}_{j} \\
0 & \text { when } \mathrm{z}_{i} \text { is less important than } \mathrm{z}_{j}
\end{array}\right.
$$

where $z_{i}$ is the index layer or criterion layer evaluation, and $z_{i} \in Z(i=1,2, \ldots, n), z_{i j}$ indicates the relative importance between $z_{i}$ and $z_{j}$.

b) Establish judgment matrix

$$
q_{i j}= \begin{cases}e_{i}\left(\frac{r_{i}-r_{j}}{r_{\max }-r_{\min }}\right) & \left(r_{i} \geq r_{j}\right) \\ 1 /\left[e_{i}\left(\frac{r_{j}-r_{i}}{r_{\max }-r_{\min }}\right)\right] & \left(r_{i}<r_{j}\right)\end{cases}
$$

where $r_{i}$ is represented as the sum of comparison matrix element in each row, $r_{\max }, r_{\min }$ signify the maximum value and the minimum value of $r_{i}$; The judgment matrix $Q=\cup q_{i j}(i, j=1,2, \ldots, n), e_{i}$ is a constant that reflects the relative importance in a certain standard. $e_{i}=9$ is often taken in practical application.

c) Calculate weight of importance

$$
\begin{gathered}
w_{i}=\sqrt[n]{\prod_{j=1}^{n} q_{i j}} \\
\bar{w}_{i}=\frac{w_{i}}{\sum_{i=1}^{n} w_{i}}
\end{gathered}
$$

By formula (9), (10), and the test of consistency, we get $\bar{w}=\left(\bar{w}_{1}, \bar{w}_{2} \ldots \bar{w}_{n}\right)^{T}$.

$$
\begin{gathered}
\bar{w}_{s r v}=\sum_{s=1}^{3} \sum_{p=1}^{n} \bar{w}_{s} \bar{w}_{p} \\
\bar{w}_{\text {host }}=\sum_{i=1}^{n} \bar{w}_{s r v_{i}}
\end{gathered}
$$

Design $\bar{w}_{s}, \bar{w}_{p}$ as index and criterion layer weight respectively. $\bar{w}_{s r v}$ is the weight of service in target layer. $\bar{w}_{\text {host }}$ is the weight of host.

\subsection{The Value of Risk Situation}

Risk situation represents the extent of harm caused by attack in the network, it can be assessed by the threat factor, the attack ability, the Attack Successful Probability, the weight of importance and other factors. As the value of risk situation increases, the danger of network also increases.

Definition 6. Risk of service (SR): within the time $(t, t+$ $\Delta t)$, the number of attack in service $S_{i}(0 \leq i \leq m)$ is $N_{i}$. Attack Successful Probability is $A P_{i}$. The number of type $k(0 \leq k \leq n)$ attack is $N_{i k}$, and it satisfies $N_{i}=\sum_{k=0}^{n} N_{i k}$. Threat factor and the attack ability of type $k$ are $T F_{k}$ and $\theta_{k}$. The risk situation of service $S_{i}$ is defined as formula (13).

$$
S R_{S_{i}}=\sum_{k=1}^{n}\left(N_{i k} 10^{T F_{i k}} \frac{\theta_{i k}+4}{7} A P_{i k}\right)
$$


Definition \%. Risk of host (HS): within the time $(t, t+\Delta t)$, $u$ kinds of services run in the host $H_{g}(1 \leq g \leq v)$ and the weight of service is $S_{i}(0 \leq i \leq u)$. The risk situation of host $H_{g}$ is defined as formula (14).

$$
H R_{H_{g}}=\sum_{i=1}^{u}\left(S R_{S_{i}} \bar{w}_{s r v_{i}}\right)
$$

Definition 8. Risk of network (NS): within the time $(t, t+$ $\Delta t), v$ kinds of hosts are running in the network and the weight of host is $H_{g}(1 \leq g \leq v)$. The risk situation of network is defined as formula (15).

$$
N R=\sum_{g=1}^{v}\left(H R_{H_{g}} \bar{w}_{\text {host }_{g}}\right)
$$

\section{EXPERIMENT RESULTS ANALYSIS AND COMPARISON}

In order to verify scientific and comprehensive of the proposed method, the simulation platform of laboratory is used to collect network data in two months from July 1, 2013 to August 31, 2013 and dynamically analyze the risk of vulnerable host.

\subsection{The Three-demarcation Analytic Hierar- chy Process to Calculate the Weight of Im- portance}

Service information and vulnerability information are used to our experiment data. The target layer expresses the importance of service; the criterion layer represents the value of the average number of visits, access frequency and degree of importance in the host; the index layer signifies the service whose importance will be calculated. By the experience, we respectively define the degree of importance, the access frequency and the average number of visits in the host for the [ $\left.\begin{array}{lll}0.6 & 0.2 & 0.2\end{array}\right]$. The host whose IP is 192.168.0.1 and exits vulnerability is taken as an example to analyze. There are four kinds of service in the host such as DNS, WWW, FTP, SMTP. The weights of criterion layers are respectively listed as $B_{1}=\left[\begin{array}{llll}0.1376 & 0.5132 & 0.2751 & 0.0741\end{array}\right], B_{2}=\left[\begin{array}{ll}0.0989 \\ 0.093\end{array}\right.$ $0.51830 .28390 .0989]$, $B_{3}=\left[\begin{array}{llll}0.0989 & 0.2839 & 0.5183 & 0.0989\end{array}\right]$. Based on this method, we can calculate the service and host weight of importance as shown in Table 3.

Through the data analysis, the three-demarcation analytic hierarchy process is not only reduces the complexity of the nine-demarcation analytic hierarchy process [6], but also avoids the fuzzy of weight when constructing the judgment matrix.

\subsection{The Threat of Attack and Attack Success- ful Probability}

By the above definition on the threat of attack and Attack Successful Probability algorithms, we take the information from service, vulnerability and attack alarm in July as the basic of data. After statistical analysis, there are 7 kinds of service in the network attacked by 12 kinds of attacks. We use the parameter estimation method in item response theory to get the attack ability and calculate Attack Successful Probability as shown in formula (4). The proposed method and the conventional methods of the threat of attack are indicated in curves of Figure 2.

In order to achieve the real-time risk situation, we count the number of attack for August each day. If there is a new

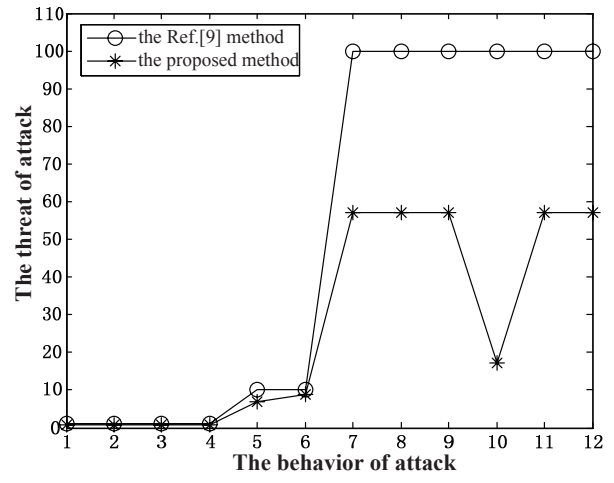

Figure 2: Comparison of the Threat of Attack with Two Methods

attack which has not been appeared last month, it will be added to the original attack response matrix. At the same time, the probability matrix and the threat of attack will be updated.

\subsection{Network Risk Assessment in Real Time}

With the data of network in August 2013, the improved risk assessment algorithm is used to calculate the risk of whole network every other day. Then, we can draw a onemonth trend curve of risk and make a decision.

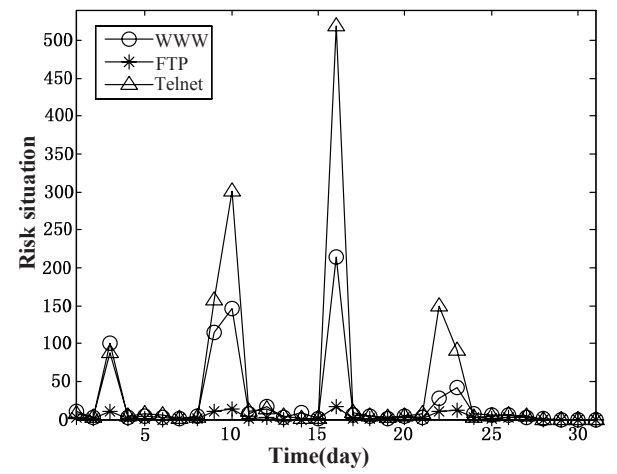

Figure 3: Risk of Services in Real Time

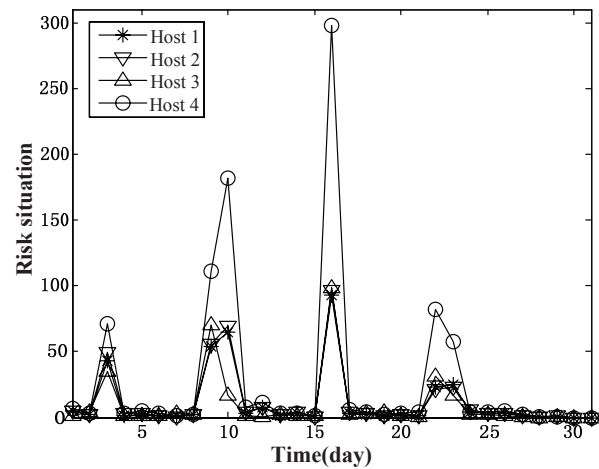

Figure 4: Risk of Hosts in Real Time 
Table 3: Network Configuration and the Distribution of the Weight

\begin{tabular}{|c|c|c|c|c|}
\hline Host IP (192.168.) & Operating System & Running Service & Service Importance Weight & Host Importance Weight \\
\hline \multirow{4}{*}{0.1} & \multirow{4}{*}{ Windows XP } & DNS & 0.10664 & \multirow{4}{*}{0.2604} \\
\hline & & WWW & 0.37664 & \\
\hline & & FTP & 0.42278 & \\
\hline & & SMTP & 0.09394 & \\
\hline \multirow{3}{*}{1.4} & \multirow{3}{*}{ Windows XP } & WWW & 0.40570 & \multirow{3}{*}{0.2339} \\
\hline & & FTP & 0.18288 & \\
\hline & & ORACLE & 0.41142 & \\
\hline \multirow{3}{*}{2.5} & \multirow{3}{*}{ Red Hat Linux } & DNS & 0.32572 & \multirow{3}{*}{0.2228} \\
\hline & & POP3 & 0.42858 & \\
\hline & & SMTP & 0.42572 & \\
\hline \multirow{5}{*}{2.7} & \multirow{5}{*}{ Windows 2000} & WWW & 0.31074 & \multirow{5}{*}{0.2829} \\
\hline & & TELNET & 0.43302 & \\
\hline & & DNS & 0.10734 & \\
\hline & & FTP & 0.08344 & \\
\hline & & ORACLE & 0.06543 & \\
\hline
\end{tabular}

Table 4: Attack Successful Probability

\begin{tabular}{|c|c|c|c|c|c|c|c|}
\hline $\begin{array}{l}\text { Attack Successful Probability Attack } \\
\text { Service }\end{array}$ & WWW & FTP & SMTP & POP3 & ORACLE & TELNET & DNS \\
\hline Ipsweep (1) & 0 & 0.1750 & 0 & 0 & 0 & 0.9801 & 0.6968 \\
\hline $\operatorname{Nmap}(2)$ & 0.9728 & 0.6256 & 0 & 0.9987 & 0.7246 & 0.9974 & 0 \\
\hline$\ldots$ & $\ldots$ & $\ldots$ & $\ldots$ & $\ldots$ & $\ldots$ & $\ldots$ & $\ldots$ \\
\hline Guess passwd(11) & 0.4945 & 0 & 0 & 0 & 0 & 0.9138 & 0 \\
\hline Warezmaster(12) & 0.4945 & 0.0437 & 0 & 0 & 0 & 0 & 0 \\
\hline
\end{tabular}

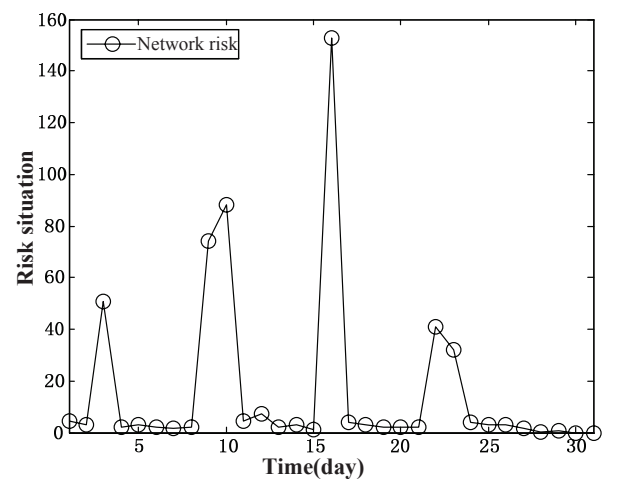

Figure 5: Risk of Network in Real Time

Figures 3, 4, 5 respectively show the variation of risk situation in service logic layer, host logical layer and network logical layer in August. The risk situation of WWW, FTP and TELNET are indicated in Figure 3. We can see the service of TELNET is the most serious attack on the month, the service of WWW Secondly and the lowest risk of attack is the service of FTP. There are four hosts which have some vulnerability in the network and the risk situation of them is exhibited in Figure 4. We know that the risk of host 4 is the largest, and the risk of the remaining three hosts is similar. The risk situation of whole network is shown in Figure 5. Through the analysis of the above three figures, network attacks are occurred, for the most part, focusing on the weekend, so we believe that the attackers are likely have

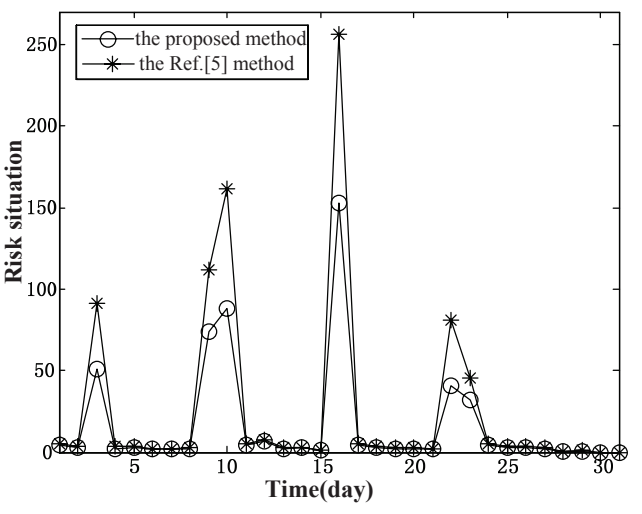

Figure 6: Comparison of Network Risk Assessment with Two Methods

legitimate careers, such as on-the-job workers or students.

\subsection{The Comparison of Network Risk}

The occurrence of network security events exist great contingency and randomness. If only considering the losses, we can hardly restore the real security situation. Thus, a novel method to assess network is proposed. The traditional assessment method of risk situation has been improved by Attack Successful Probability, the weight of importance, Service Security Level and the attack ability. The network risk assessment results of two different methods are indicated in curves of Figure 6. It is obvious that the value of risk 
situation in method [5] is higher than that of the proposed method. In fact, some attacks do not successfully harm to the network and the calculation of the threat of attack is imprecise. For example, a few days with high differentiation in security could have used several different decisions on network protection, but the gap between the results from conventional methods may not be obvious and use the same decision. So we can reduce the waste of resources by the proposed method. These issues will be easy to cause the phenomenon that the conventional methods can not reflec$\mathrm{t}$ the real status of network security, thus misleading the network security administrator, or even making the wrong decision. Accordingly with the method for risk assessment in this paper, quantitative risk situation is not only obtained, but also comprehensive and directive.

\section{CONCLUSION}

In this paper, we have quantitatively analyzed the risk situation in service, host and network logic layer, and proposed a novel risk assessment method for network security based on item response theory. Compared with traditional methods, the advantages are exhibited as follow:

1) In contrast with the risk assessment by vulnerability coming from security detecting and scanning facilities, we have defined an assessment of security parameter by the combination of CVSS with Defensive intensity, so we can get more comprehensive and reasonable results.

2) Through the three-demarcation analytic hierarchy process to improve the accuracy of the importance weight.

3) Combining with item response theory, we propose a higher degree of discrimination in the threat of attack algorithm and a more realistic Attack Successful Probability algorithm.

4) With the Attack Successful Probability and the threat of attack to update dynamically, we realize the real-time assessment of risk situation.

Due to the affected by the accuracy of the dataset, this method has certain limitations. The next work will be devoted to improving the accuracy of the dataset in the largescale network. We would like to obtain more comprehensive and more precise quantitative risk situation analysis.

\section{ACKNOWLEDGMENTS}

This work was partially supported by the National Natural Science Foundation of China (NO.61271260, NO.61301122) and the Science and Technology Research Project of Chongqing Education Commission (NO.KJ120530).

\section{REFERENCES}

[1] M. Abedin, S. Nessa, and E. Al-Shaer. Vulnerability analysis for evaluating quality of protection of security policies. In Proceedings of the 2nd ACM workshop on Quality of protection, pages 49-52, 2006.

[2] A. Ali, P. Zavarsky, D. Lindskog, and R. Ruhl. A software application to analyze the effects of temporal and environmental metrics on overall cvss v2 score. In Internet Security (WorldCIS), 2011 World Congress on, pages 109-113, 2011.
[3] F. Arnold, W. Pieters, and M. Stoelinga. Quantitative penetration testing with item response theory. In Information Assurance and Security (IAS), 2013 9th International Conference on, pages 49-54, 2013.

[4] S. Baldiris, R. Fabregat, and S. Graf. Learning object recommendations based on quality and item response theory. In Advanced Learning Technologies (ICALT), 2014 IEEE 14th International Conference on, pages 34$36,2014$.

[5] X. Z. Chen, Q. H. Zheng, and X. H. Guan. Quantitative hierarchical threat evaluation model for network security. Journal of Software, 17(4):885-897, 2006.

[6] S. Fu and H. J. Zhou. The information security risk assessment based on ahp and fuzzy comprehensive evaluation. In Communication Software and Networks (ICCSN), 2011 IEEE 3rd International Conference on, pages 124-128, 2011.

[7] Z. H. Gong and Y. Zhou. Research on cyberspace situational awareness. Journal of Software, 21(7):1605-1619, 2010 .

[8] M. Keramati, A. Akbari, and M. Keramati. Cvssbased security metrics for quantitative analysis of attack graphs. In Computer and Knowledge Engineering (ICCKE), 2013 3th International eConference on, pages 178-183, 2013.

[9] G. Liu, Q. M. Li, and H. Zhang. Reliability vector orthogonal projection decomposition method of network security risk assessment. Journal of Electronics and Information Technology, 34(8):1934-1938, 2012.

[10] H. Tsai and Y. L. Huang. An analytic hierarchy processbased risk assessment method for wireless networks. Reliability, IEEE Transactions on, 60(4):801-816, 2011.

[11] H. Wang, J. Chen, and C. Q. Ma. Research on the improvement of irt item parameter estimation algorithm. In Computational Intelligence and Design (ISCID), 2012 Fifth International Symposium on, volume 1, pages 160-163, 2012.

[12] X. H. Wang and L. P. Zou. A decision model for agile software release. In Reliability, Maintainability and Safety (ICRMS), 2011 9th International Conference on, pages 766-770, 2011.

[13] Y. Wei, Y. F. Lian, and D. G. Feng. A network security situational awareness model based on information fusion. Journal of Computer Research and Development, 46(3):353-362, 2009. 